\title{
Effect of Lysyllysine on non-covalent hybridization of single walled carbon nanotube by single-stranded DNA homodimer: in silico approach
}

\author{
Fateme Bagherolhashemi $^{1} \cdot$ Mohammad Reza Bozorgmehr $^{1}$ (D) $\cdot$ Mohammad Momen-Heravi $^{1}$
}

Received: 1 June 2019 / Accepted: 29 October 2019 / Published online: 7 November 2019

(c) The Author(s) 2019

\section{Abstract}

In this work, the interactions between adenine-adenine di-nucleotide (DA2N) and carbon nanotube (CNT) in the presence of Lysyllysine (LL) was studied by the molecular dynamics simulation. Different carbon nanotubes including (5.5), (6.6) and (7.7) were used to investigate the effect of CNT type. The binding energies were calculated using the molecular mechanicsPoisson Bolzmann surface area method. The results showed that the contribution of the van der Waals interactions between DA2N and CNT was greater than that of the electrostatic interactions. The LL significantly enhanced the electrostatic interactions between the DA2N and CNT (6.6). The quantum calculations revealed that the sensor properties of the DA2N were not significantly affected by the CNT and LL. However, the five-membered ring of adenine played a more important role in the sensing properties of the DA2N. The obtained results are consistent with the previous experimental observations that can help to understand the molecular mechanism of the interaction of DA2N with CNT.

\section{Graphic abstract}

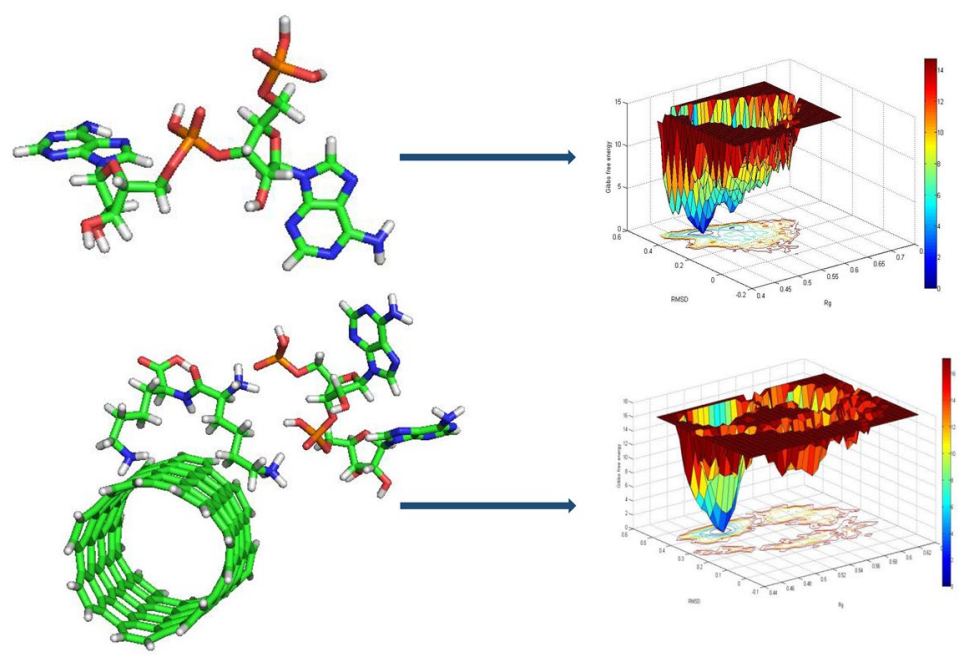

Electronic supplementary material The online version of this article (https://doi.org/10.1007/s40097-019-00320-1) contains supplementary material, which is available to authorized users.

Mohammad Reza Bozorgmehr

mr_bozorgmehr@yahoo.com; bozorgmehr@mshdiau.ac.ir

Extended author information available on the last page of the article 


\title{
Highlights
}

- LL significantly enhanced the electrostatic interactions between the DA2N and CNT.

- Sensor properties of the DA2N were not significantly affected by the CNT and LL.

- The contribution of the van der Waals interactions was greater than that of the electrostatic interactions.

\author{
Keywords Adenine $\cdot$ Biosensor $\cdot$ Lysine $\cdot$ Aromatic
}

\section{Introduction}

Polymerase chain reaction (PCR) and biochip are two conventional techniques for the identification of the transgenic food products [1, 2]. In the PCR technique, due to the nucleic acid recognition sequence of a gene and its function, large quantities $(\mu \mathrm{g})$ of a particular nucleotide sequence are proliferated [3]. This method involves three steps as denaturation of deoxyribonucleic acid (DNA), binding primers to the DNA sequence, and polymerization [4]. On the other hand, the biochip, which is a semiconductor, is prepared based on the DNA structure [5]. The biochip method is the basis of the chemical analyses, including the flow injection analysis and the identification of molecules [6]. Although there are many applications for the PCR and biochip methods, they suffer from some drawbacks such as the contamination of the samples examined by the external DNA fragments [7, 8]. Therefore, DNA-based electrochemical biosensors have been introduced as new methods with higher sensitivity, smaller dimension, and lower cost $[9,10]$. Hence, in many cases, these biosensors have been used for the identification of the transgenic products [11, 12]. For instance, Ren et al. prepared a DNA-based electrochemical biosensor with the immobilization of DNA on aluminum ion films [13]. They electrodeposited this biosensor on the surface of a modified carbon paste electrode and used it successfully to detect the transgenic soybeans. Jiao et al. prepared a DNA-based electrochemical biosensor on the surface of the gold nanoparticles, using the modified poly(2,6-pyridinedicarboxylic acid) film, for the identification of the phosphinothricin (Pt) $\mathrm{N}$-acetyltransferase gene (PAT) in the transgenic crops with the detection limit of $2.4 \times 10^{-11} \mathrm{~mol} / \mathrm{L}$ [14]. The key step for providing the DNA-based biosensors is the immobilization of DNA on different surfaces [15]. This step determines the stability, repeatability, recyclability and sensitivity of the biosensors. In overall, in the preparation of biosensors, it is important to maintain the stability, reproducibility and recoverability of the biological agent. For this reason, biological identifiers are immobilized on a support surface. On the other hand, the support surface must be neutral so as not to affect the activity of the biological agent. Therefore, carbon nanotubes are suitable candidates for this purpose. In addition, in order to maintain the stability of the identifier, it is important to select the appropriate size of CNT for the stabilizing surface. Also, because biological nanotubes are non-polar and do not have functional groups, it is difficult to stabilize biological agents on their surface. Therefore, nanotube functionalization is used to solve this problem. The nanotube functionalization is done in both covalent and non-covalent methods. In the covalent functionalization method, the nature of the nanotube changes and it is difficult to control its effect on the detecting agent. In non-covalent functionalization, the functional group must interact with the nanotube on the one hand and the identifier on the other. Various functional groups, such as those in poly-L-lysine, have been used to immobilize the DNA on different surfaces [16]. Poly-L-lysine has positively charged side chains, and therefore, can have appropriate interactions with the negatively charged phosphate groups of DNA. On the other hand, carbon nanotubes (CNT), with high specific surface areas, are appropriate surfaces for these DNA biosensors to be immobilized on $[17,18]$. The biosensors immobilized on the CNT surface using poly-L-lysine have been extensively studied [19, 20]. However, the interaction of DNA and CNT with a focus on the role of poly-L-lysine has not been studied. Therefore, in this study, the role of Lysyllysine (LL) in the interaction of DA2N with three types of carbon nanotubes, i.e., $(5,5),(6,6)$, and $(7,7)$, was studied using molecular dynamics simulation.

\section{Computational details}

To construct the primary dinucleotide DA2N structure from the $\mathrm{B}$ type DNA (B-DNA), the 1BNA code in the protein data bank (PDB) was used [21]. Then, using this dinucleotide, the DA2N was designed in silico by Pymol software [22]. Eight simulation boxes with the dimensions of $4 \times 4 \times 4 \mathrm{~nm}^{3}$ were designed and the DA2N nucleotide was placed in the center of all the boxes. Then, the armchairs CNT $(5,5), \operatorname{CNT}(6,6)$, and CNT $(7,7)$ with $(15.11,7.72),(15.13,7.74)$ and $(15.14,7.75)$ dimension (in angstrom), respectively were placed in three of these boxes. In other three boxes, in addition to the CNTs the LL was also included. Also, a box was assigned to the DA2N and LL, and another box to the DA2N only. The force field parameters of the DA2N and CNT are not by default found in the Gromacs software, and therefore, the SwissParam website was used [23]. The structures were optimized using 
Gaussian03 software at the HF/6-31G level of theory [24]. The partial atomic charges were obtained from the CHELPG method [25]. To ensure optimization of structures, frequency calculations were performed using the same method and the same previous function. The results showed that there are no virtual frequencies for the optimal structures. The calculations of this step were done using the GAMESS software. Each simulation box was filled with TIP3P water [26]. Since the net charge of the designed systems is not zero, some sodium cations were added to each box to neutralize them. For the simulations, at first, the energy minimization was carried out using the steepest descent algorithm. Each simulation box achieved two-stage equilibrium in NVT and NPT ensemble. At this stage, the time of equilibration was considered to be 1000 ps with the time step 2 fs. Finally, molecular dynamics was performed for 10000 ps with the 2 fs time step. Each simulation was repeated four times under the different initial conditions to avoid any dependency on the initial conditions and to increase the accuracy of the simulations. Therefore, the total time reaches $\sim 320 \mathrm{~ns}$ for long MD simulation. The PME algorithm was used to calculate electrostatic interactions [27]. LINCS algorithm [28] was employed to fix the chemical bonds between the atoms of the protein. In the case of solvent molecules the SETTLE algorithm was used [29]. The systems components were coupled with V-rescale and Nose-Hoover thermostat to fix a constant temperature and pressure, respectively during the simulations for each equilibration steps [30]. The simulations were carried out with Gromacs 5.1.2 [31] using the CHARMM-AA force field [32].

\section{Results and discussion}

To evaluate the binding affinities between the DA2N and CNT in the presence of LL, the molecular mechanicsPoisson Bolzmann surface area (MMPBSA) algorithm,

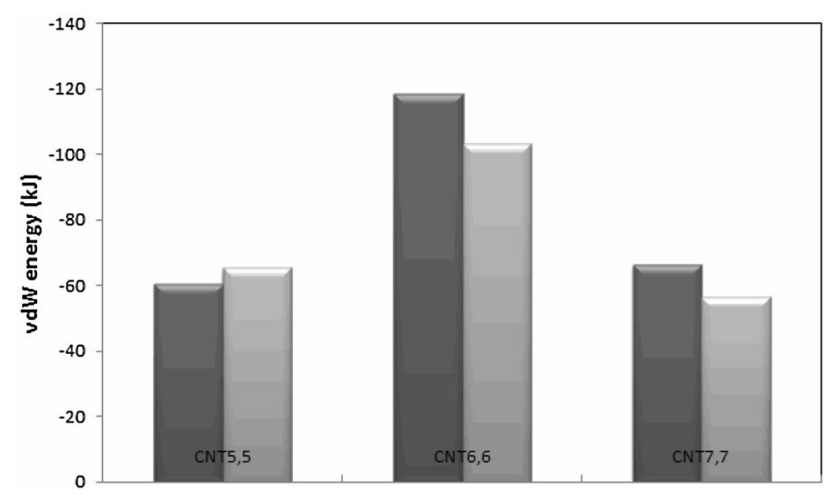

Fig. 1 vdW energy contributions of interaction between DA2N and CNT $\mathbf{a}$ in presence of LL (solid dark color) $\mathbf{b}$ in absence of LL (lightdark color) first presented by Kollman et al., was used [33, 34]. In this method, the free energy is obtained by:

$G=E_{\mathrm{bnd}}+E_{\mathrm{el}}+E_{\mathrm{vdW}}+G_{\mathrm{pol}}+G_{\mathrm{np}}-T S$,

where $E_{\mathrm{bnd}}, E_{\mathrm{el}}$ and $E_{\mathrm{vdW}}$ are the MM energies (bonding, bending, and dihedral), electrostatic energy and van der Waals interactions, respectively; $G_{\mathrm{pol}}$ and $G_{\mathrm{np}}$ are polar and nonpolar solvation free energies, which were obtained from the generalized Born and solvent accessible surface methods, respectively. The last term in Eq. (1), in which $T$ is temperature and $S$ is entropy, is obtained from the normal mode (NM) analysis. The contribution of van der Waals and electrostatic interactions along with the binding free energy for the DA2N and CNT system in the presence and absence of LL (Figs. 1, 2, 3) was calculated using Eq. (1). In the Figs. 1, 2 and 3, the bright gray and dark colors are associated with the absence and presence of LL, respectively.

Accordingly, the contribution of the van der Waals interactions is higher than that of the electrostatic interactions. Also,

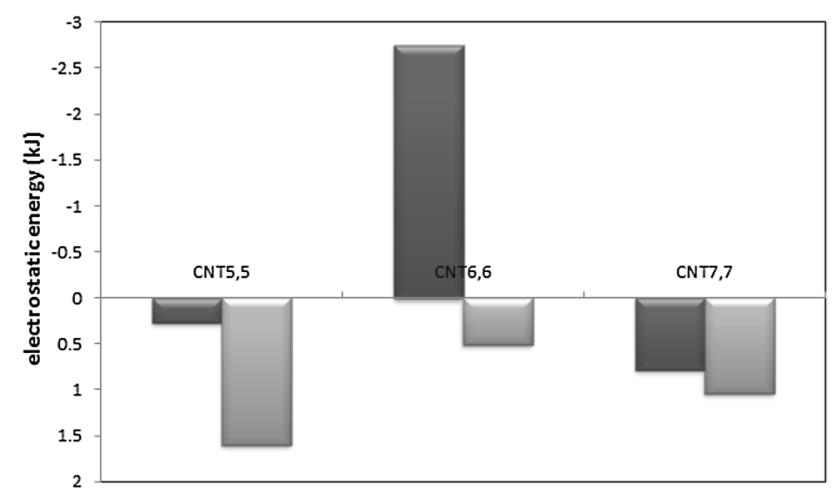

Fig. 2 Electrostatic energy contributions of interaction between DA2N and CNT $\mathbf{a}$ in presence of LL (solid dark color) $\mathbf{b}$ in absence of LL (light-dark color)

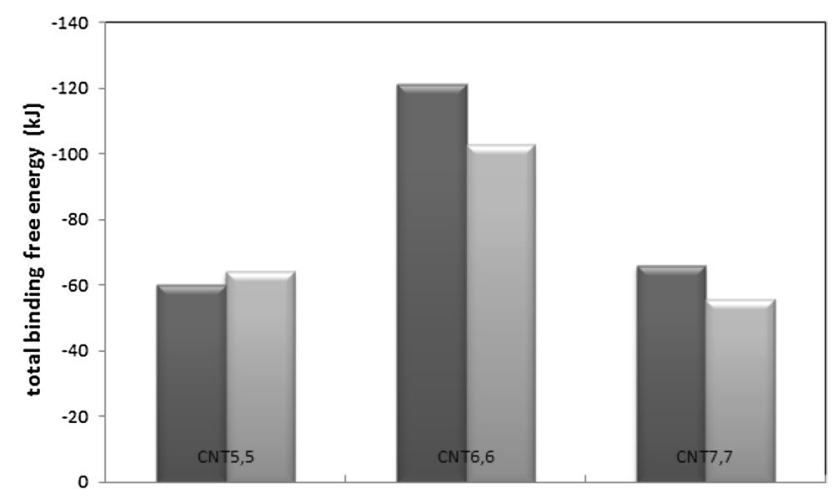

Fig. 3 Total Gibbs free energy of interaction between DA2N and CNT $\mathbf{a}$ in presence of LL (solid dark color) $\mathbf{b}$ in absence of LL (lightdark color) 
the presence of LL shows a different role on the interactions between DA2N and CNT. For instance, in the presence of LL the vdW interactions decrease for the CNT $(5,5)$, while it enhances for the CNTs $(6,6)$ and $(7,7)$. The highest vdW interactions can be seen in CNT $(6,6)$ system. Furthermore, the presence of $\mathrm{LL}$ is desirable for the electrostatic interactions between the DA2N and for CNT $(6,6)$, whereas it is unfavorable for the CNT $(5,5)$ and CNT $(7,7)$. In fact, the LL leads to the enhancement of the DA2N binding affinity to the CNT $(6,6)$. Summary results obtained from Figs. 1 to 3 are listed in Table 1. In this table, decrease or increase of the types of interaction energies due to the presence of LL in the studied systems has been reported. Depending on the orientation of the LL between the nanotubes and the nucleic acid, the type of intermolecular forces will be affected. By observing snapshots of the simulated systems in the last $1 \mathrm{~ns}$, it was found that in the case of the interaction of the CNT $(5,5)$ with the nucleic acid, the LL had a more coating effect between the CNT and the nucleic acid. Thus, by reducing the van der Waals contact area between these species reduced van der Waals interactions. Whereas, in the case of nanotubes $(6,6)$ and $(7,7), \mathrm{LL}$ is not completely located between the nucleic acid and the nanotubes. These findings are consistent with the results reported in previous work [35]. However, it should be noted that the MMPBSA method does not take into account the electronic structure effects, so the absolute amounts of binding energies obtained cannot be invoked. The free energy landscape (FEL) analysis have been used for further interpretation of the results [36]. One of the important issues in the molecular dynamics simulations is the sampling of the structures suitable for analysis. Different methods, such as averaging over the last several nanoseconds of simulation, and averaging from where the system is in equilibrium, have been used for sampling. In the latter case, variations of a quantity such as RMSD are used for determining the equilibrium region. The averaging over the atomic coordinates does not have a physical meaning, and therefore, the FEL analysis was used. In this analysis, the RMSD and DA2N radius of gyration $\left(R_{\mathrm{g}}\right)$ were used in each simulation. The root mean square deviation (RMSD) is defined as:

$\operatorname{RMSD}\left(t_{1}, t_{2}\right)=\left[\frac{1}{M} \sum_{i=1}^{N} m_{i}\left\|r_{i}\left(t_{1}\right)-r_{i}\left(t_{2}\right)\right\|\right]^{\frac{1}{2}}$, where $r_{i}$ is the atomic position at time $t$ and $M=\sum_{i=1}^{N} m_{i}$. When the RMSD takes a flat shape, it is a sign of the equilibrium of the system, and everywhere in the RMSD that there are more fluctuations; it is a sign of a change in molecule structure in which this change may be caused by the opening or compressing of the structure. Also, the $R_{\mathrm{g}}$ value of protein is calculated using following relation:

$R_{\mathrm{g}}=\left(\frac{\sum_{i}\left\|r_{i}\right\|^{2} m_{i}}{\sum_{i} m_{i}}\right)^{\frac{1}{2}}$,

where $m_{i}$ is the atomic mass of $i$ and $r_{i}$ the atomic position of $i$ relative to the center of the molecule. Then, the probability of different DA2N conformations was calculated in respect to the selected variables. The obtained results in terms of three-dimensional diagrams are shown in Fig. 4 and Figs. 1S to 7S (in support information data). Accordingly, the regions coincident with the minimum and maximum free energies are shown in blue and red, respectively. Also, the values of RMSD and $R_{\mathrm{g}}$ corresponded to the minimum free energy was calculated for the DA2N in each simulation, and the results are reported in Table 2. As clear in Fig. 4 and Figs. 1S to 7S and Table 2, the LL in the presence of CNT $(6,6)$ results in the DA2N stability. According to the information reported in Table 2, it is observed that the presence of LL increased the $R_{\mathrm{g}}$ of DA2N. In other word, a more open conformation of DA2N is created in the presence of LL. Since molecular dynamics calculations do not take into account electron motions, it is not possible to investigate the DA2N sensor properties by this method alone. For this purpose, quantum computations were performed on structures sampled from molecular dynamics simulation. A single point calculation at the HF/6-31G level was performed on the FEL-sampled DA2N to investigate the effect of the LL on the sensing capability of the DA2N. Then, the Multiwfn software [37] was used to calculate the total density of state (TDOS) and partial density of state (PDOS) (Fig. 5 and Figs. $8 \mathrm{~S}$ to $13 \mathrm{~S}$ in support information data). The five-membered and six-membered rings of adenine were indexed to calculate the PDOS. In Fig. 5 and Figs. 8S to $13 \mathrm{~S}$ in support information data, the dark, red, and blue colors are related to the TDOS, PDOS of the six-membered
Table 1 Decrease or increase of the types of interaction energy due to the presence of LL in the studied systems

\begin{tabular}{llll}
\hline Kind of energy & Designed system & & \\
\cline { 2 - 4 } & DA2N+CNT $(5,5)$ & DA2N +CNT $(6,6)$ & DA2N + CNT (7, 7) \\
\hline Van der Walls & Decrease & Increase & Increase \\
Electrostatic & Decrease & Increase & Decrease \\
Total binding energy & Decrease & Increase & Increase \\
\hline
\end{tabular}




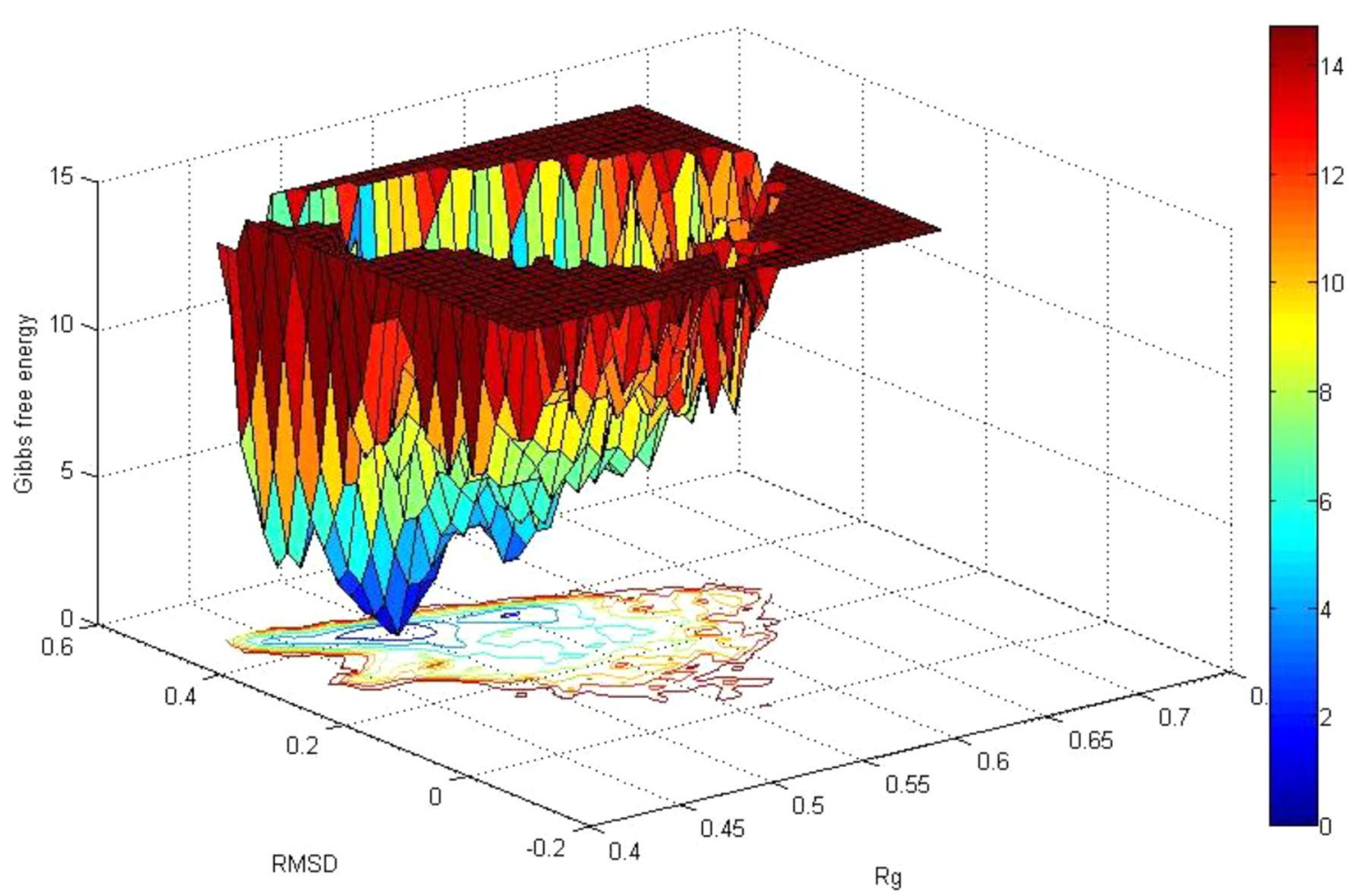

Fig. 4 Free energy landscape of the DA2N

Table 2 Radius of gyration along with RMSD values of DA2N for different simulated systems obtained by the FEL sample analysis

\begin{tabular}{lll}
\hline Simulated system & $\begin{array}{l}\text { Radius of gyration } \\
(\mathrm{nm})\end{array}$ & RMSD $(\mathrm{nm})$ \\
\hline DA2N & 0.4931 & 0.3888 \\
DA2N + LL & 0.4604 & 0.3303 \\
DA2N + CNT $(5,5)$ & 0.5527 & 0.1974 \\
DA2N + CNT $(6,6)$ & 0.5006 & 0.3945 \\
DA2N + CNT $(7,7)$ & 0.4619 & 0.2347 \\
DA2N + CNT $(5,5)+$ LL & 0.4997 & 0.2014 \\
DA2N + CNT $(6,6)+$ LL & 0.4996 & 0.1892 \\
DA2N + CNT $(7,7)+$ LL & 0.5799 & 0.2578 \\
\hline
\end{tabular}

ring, and PDOS of the five-membered ring, respectively. The dashed line shows the boundary between the valence and conduction bands. The total density of states (TDOS) obtained from the energy eigen values is the most insightful information in revealing the interactions and the size of the highest occupied molecular orbital (HOMO)-lowest unoccupied molecular orbital (LUMO) gap in complex multicomponent materials. The TDOS is further resolved into the partial density of states (PDOS) for each atomic species or each structural component. It is seen that the contribution of the five-membered ring in all systems is more than that of the six-membered ring. However, the overall patterns of TDOS and PDOS in all studied systems do not show a significant change, which is indicative of the fact that the electron transfer is not significant in the DA2N in the presence of the LL. In other words, the sensing properties of the DA2N have not undergone significant changes by its complexation with CNT. In fact, as stated in other Ref. [38], the overall features of the TDOS are quite similar to other biomolecular systems since they all consist mainly of $\mathrm{C}, \mathrm{O}, \mathrm{N}, \mathrm{P}$ and $\mathrm{H}$ atoms with modifications based on detailed structures and additional component atoms. The results show good agreement with experimental results. Electrochemically investigation of the interaction of calf thymus double stranded DNA with 4,4'-dihydroxy chalcone show that signal detection is related to oxidation of adenine. The oxidation of adenine is attributed to its nitrogen atoms, in particular the nitrogen atom of the five-membered ring of adenine [39]. In other experimental work, a dsDNA-electrochemical biosensor was use for the in situ evaluation of some heavy ions such as $\mathrm{Ni}^{2+}$, $\mathrm{Pb}^{2+}$ and $\mathrm{Cd}^{2+}$. The authors have been shown that oxidation peaks of adenosine bases changes in presence of ions; these change preferentially occurred at adenine-containing rings [40]. The average structure of the LL was obtained from the 100 final frames of the simulations. Single point energy calculations were performed for average structures of LL with the same level of theory and basis set as described in 


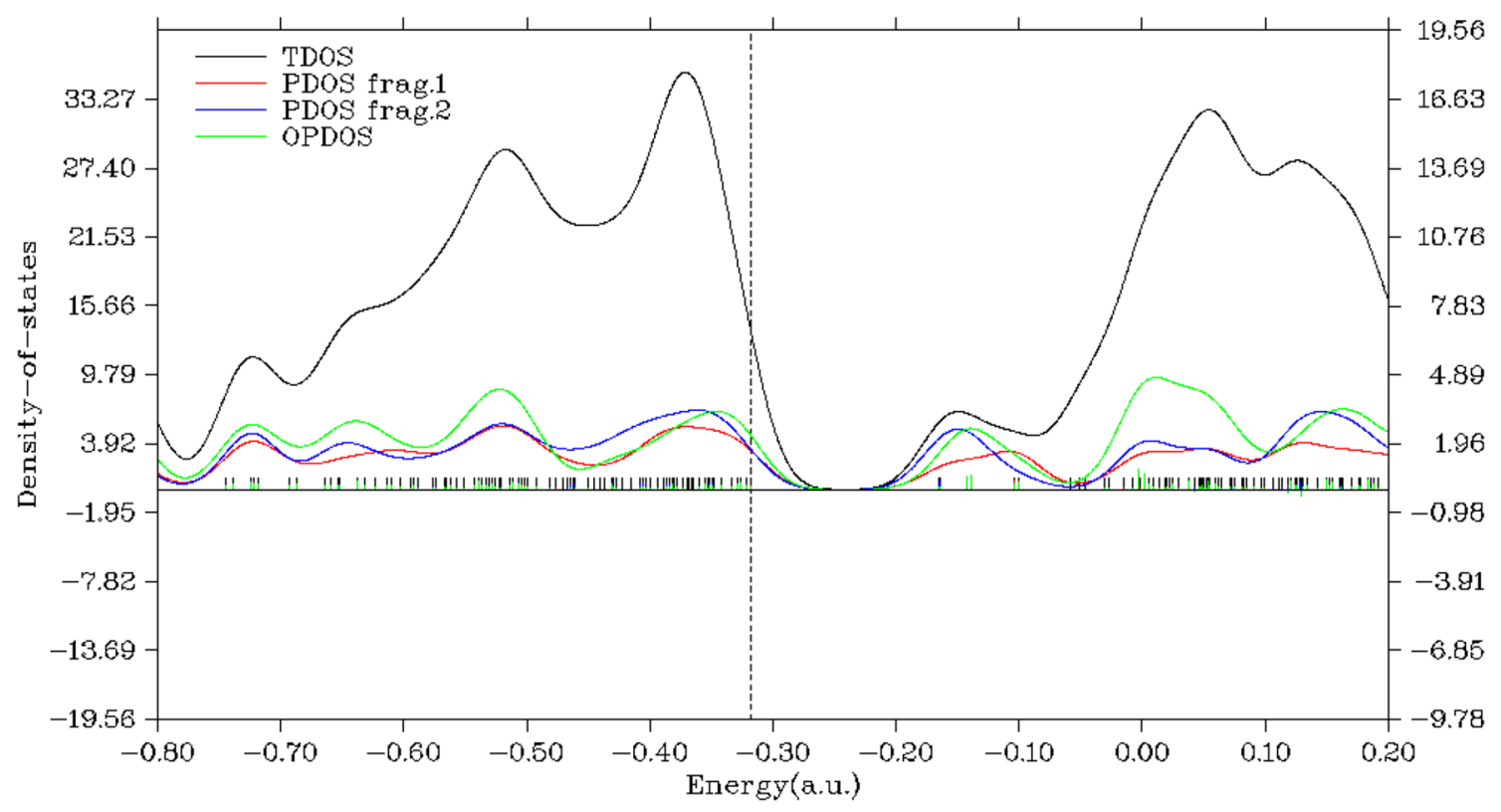

Fig. 5 Diagram of TDOS, PDOS and OPDOS for DA2N. The frag.1 and frag.2 are six-membered and five-membered rings of DA2N, respectively

the method section. The HOMO and the LUMO of LL were obtained in different simulations and are shown in Fig. 14S. According to the figure, the location of the orbitals 1 and 2 for the peptide is almost identical in all simulations, indicating that the peptide does not have a reactive role in the biosensor but acts as a linker between the nanotube and the nucleic acid.

\section{Conclusions}

Here, using the molecular dynamics simulation and quantum mechanics, the mechanism of interaction between these three components was investigated. The results of the molecular dynamics simulation showed that with the increase in the electrostatic forces, the stabilization of the DA2N on the CNT is improved. However, in presence and absence of LL, van der Waals forces play a key role in the interaction between DA2N and CNT. Also, the type of CNT plays a role in the interaction between these two species, as the interaction of the DA2N with CNT $(6,6)$ is greater than that of the $(5,5)$ and $(7,7)$ CNTs. The structural geometry figure of system containing CNT $(6,6)$, LL and DA2N was shown in supporting information file (Fig. 15S). The results from quantum computation showed that the presence of LL does not change the biosensor properties of the DA2N, but improves its immobilization on the CNT. Adenine is a purine consists of a six-membered nitrogen ring fused to a fivemembered nitrogen ring. The results from quantum computing also showed that the five-membered ring of adenine plays a larger role in its sensor properties.
Open Access This article is distributed under the terms of the Creative Commons Attribution 4.0 International License (http://creativeco mmons.org/licenses/by/4.0/), which permits unrestricted use, distribution, and reproduction in any medium, provided you give appropriate credit to the original author(s) and the source, provide a link to the Creative Commons license, and indicate if changes were made.

\section{References}

1. Deisingh, A.K., Badrie, N.: Detection approaches for genetically modified organisms in foods. Food Res. Int. 38(6), 639-649 (2005)

2. Ahmed, F.E.: Detection of genetically modified organisms in foods. Trends Biotechnol. 20(5), 215-223 (2002)

3. Ge, X., Wu, Q., Jung, Y.-C., Chen, J., Wang, S.M.: A large quantity of novel human antisense transcripts detected by LongSAGE. Bioinformatics 22(20), 2475-2479 (2006)

4. Cha, R.S., Thilly, W.G.: Specificity, efficiency, and fidelity of PCR. PCR Methods Appl. 3(3), 18-29 (1993)

5. Campas, M., Katakis, I.: DNA biochip arraying, detection and amplification strategies. Trends Anal. Chem. 23(1), 49-62 (2004)

6. Vo-Dinh, T.: Development of a DNA biochip: principle and applications. Sens. Actuators B Chem. 51(1), 52-59 (1998)

7. Postollec, F., Falentin, H., Pavan, S., Combrisson, J., Sohier, D.: Recent advances in quantitative PCR (qPCR) applications in food microbiology. Food Microbiol. 28(5), 848-861 (2011)

8. Zhang, C., Xing, D.: Miniaturized PCR chips for nucleic acid amplification and analysis: latest advances and future trends. Nucleic Acids Res. 35(13), 4223-4237 (2007)

9. Drummond, T.G., Hill, M.G., Barton, J.K.: Electrochemical DNA sensors. Nat. Biotechnol. 21(10), 1192-1199 (2003)

10. Wang, J., Rivas, G., Cai, X., Palecek, E., Nielsen, P., Shiraishi, H., Dontha, N., Luo, D., Parrado, C., Chicharro, M.: DNA electrochemical biosensors for environmental monitoring. A review. Analytica Chimica Acta 347(1-2), 1-8 (1997) 
11. Kalogianni, D.P., Koraki, T., Christopoulos, T.K., Ioannou, P.C.: Nanoparticle-based DNA biosensor for visual detection of genetically modified organisms. Biosens. Bioelectron. 21(7), 1069-1076 (2006)

12. Bogani, P., Minunni, M., Spiriti, M.M., Zavaglia, M., Tombelli, S., Buiatti, M., Mascini, M.: Transgenes monitoring in an industrial soybean processing chain by DNA-based conventional approaches and biosensors. Food Chem. 113(2), 658-664 (2009)

13. Ren, Y., Jiao, K., Xu, G., Sun, W., Gao, H.: An electrochemical DNA sensor based on electrodepositing aluminum ion films on stearic acid-modified carbon paste electrode and its application for the detection of specific sequences related to bar gene and CP4 Epsps gene. Electroanalysis 17(23), 2182-2189 (2005)

14. Yang, J., Yang, T., Feng, Y., Jiao, K.: A DNA electrochemical sensor based on nanogold-modified poly-2,6-pyridinedicarboxylic acid film and detection of PAT gene fragment. Anal. Biochem. 365(1), 24-30 (2007)

15. Zhai, J., Cui, H., Yang, R.: DNA based biosensors. Biotechnol. Adv. 15(1), 43-58 (1997)

16. Sukhorukov, G.B., Montrel, M.M., Petrov, A.I., Shabarchina, L.I., Sukhorukov, B.I.: Multilayer films containing immobilized nucleic acids. Their structure and possibilities in biosensor applications. Biosens Bioelectron. 11(9), 913-922 (1996)

17. Wang, S., Wang, R., Sellin, P., Zhang, Q.: DNA biosensors based on self-assembled carbon nanotubes. Biochem. Biophys. Res. Commun. 325(4), 1433-1437 (2004)

18. Merkoçi, A., Pumera, M., Llopis, X., Pérez, B., del Valle, M., Alegret, S.: New materials for electrochemical sensing VI: carbon nanotubes. Trends Anal. Chem. 24(9), 826-838 (2005)

19. Jiang, C., Yang, T., Jiao, K., Gao, H.: A DNA electrochemical sensor with poly-L-lysine/single-walled carbon nanotubes films and its application for the highly sensitive EIS detection of PAT gene fragment and PCR amplification of NOS gene. Electrochim. Acta 53(6), 2917-2924 (2008)

20. Wang, J., Zhang, S., Zhang, Y.: Fabrication of chronocoulometric DNA sensor based on gold nanoparticles/poly (L-lysine) modified glassy carbon electrode. Anal. Biochem. 396(2), 304-309 (2010)

21. Drew, H.R., Wing, R.M., Takano, T., Broka, C., Tanaka, S., Itakura, K., Dickerson, R.E.: Structure of a B-DNA dodecamer: conformation and dynamics. Proc. Natl. Acad. Sci. 78(4), 2179$2183(1981)$

22. De Lano, W.L.: Pymol: an open-source molecular graphics tool. CCP4 Newsl. Protein Crystallogr. 40, 82-92 (2002)

23. Zoete, V., Cuendet, M.A., Grosdidier, A., Michielin, O.: SwissParam: a fast force field generation tool for small organic molecules. J. Comput. Chem. 32(11), 2359-2368 (2011)

24. Frisch, M., Trucks, G., Schlegel, H., Scuseria, G., Robb, M., Cheeseman, J., Montgomery, J., Vreven, T., Kudin, K., Burant, J.: Gaussian 03, revision C. 02. (2008)

25. Breneman, C.M., Wiberg, K.B.: Determining atom-centered monopoles from molecular electrostatic potentials. The need for high sampling density in formamide conformational analysis. J. Comput. Chem. 11(3), 361-373 (1990)

26. Price, D.J., Brooks III, C.L.: A modified TIP3P water potential for simulation with Ewald summation. J. Chem. Phys. 121(20), 10096-10103 (2004)
27. Essmann, U., Perera, L., Berkowitz, M.L., Darden, T., Lee, H., Pedersen, L.G.: A smooth particle mesh Ewald method. J. Chem. Phys. 103(19), 8577-8593 (1995)

28. Hess, B., Bekker, H., Berendsen, H.J., Fraaije, J.G.: LINCS: a linear constraint solver for molecular simulations. J. Comput. Chem. 18(12), 1463-1472 (1997)

29. Miyamoto, S., Kollman, P.A.: Settle: an analytical version of the SHAKE and RATTLE algorithm for rigid water models. J. Comput. Chem. 13(8), 952-962 (1992)

30. Bussi, G., Donadio, D., Parrinello, M.: Canonical sampling through velocity rescaling. J. Chem. Phys. 126(1), 014101 (2007)

31. Van Der Spoel, D., Lindahl, E., Hess, B., Groenhof, G., Mark, A.E., Berendsen, H.J.: GROMACS: fast, flexible, and free. J. Comput. Chem. 26(16), 1701-1718 (2005)

32. MacKerell. A.D. Jr: Atomistic models and force fields. In: Computational Biochemistry and Biophysics, pp. 7-38 (2001)

33. Kumari, R., Consortium O.S.D.D., Lynn, A.: g_mmpbsa A GROMACS tool for high-throughput MM-PBSA calculations. J. Chem. Inf. Model. 54(7), 1951-1962 (2014)

34. Kollman, P.A., Massova, I., Reyes, C., Kuhn, B., Huo, S., Chong, L., Lee, M., Lee, T., Duan, Y., Wang, W.: Calculating structures and free energies of complex molecules: combining molecular mechanics and continuum models. Acc. Chem. Res. 33(12), 889897 (2000)

35. Bagherolhashemi, F., Bozorgmehr, M., Momen-Heravi, M.: Biosensor properties of DA-DA dinucleotide in the presence of DIL-lysine and single carbon nanotubes: molecular dynamics simulation and density functional theory approach. J. Struct. Chem. 59(5), 1228-1235 (2018)

36. Lei, H., Wu, C., Liu, H., Duan, Y.: Folding free-energy landscape of villin headpiece subdomain from molecular dynamics simulations. Proc. Natl. Acad. Sci. 104(12), 4925-4930 (2007)

37. Lu, T., Chen, F.: Multiwfn: a multifunctional wavefunction analyzer. J. Comput. Chem. 33(5), 580-592 (2012)

38. Poudel, L., Steinmetz, N.F., French, R.H., Parsegian, V.A., Podgornik, R., Ching, W.-Y.: Implication of the solvent effect, metal ions and topology in the electronic structure and hydrogen bonding of human telomeric G-quadruplex DNA. Phys. Chem. Chem. Phys. 18(31), 21573-21585 (2016)

39. Meric, B., Kerman, K., Ozkan, D., Kara, P., Erdem, A., Kucukoglu, O., Erciyas, E., Ozsoz, M.: Electrochemical biosensor for the interaction of DNA with the alkylating agent $4,4^{\prime}$-dihydroxy chalcone based on guanine and adenine signals. J. Pharm. Biomed. Anal. 30(4), 1339-1346 (2002)

40. Oliveira, S., Corduneanu, O., Oliveira-Brett, A.: In situ evaluation of heavy metal-DNA interactions using an electrochemical DNA biosensor. Bioelectrochemistry 72(1), 53-58 (2008)

Publisher's Note Springer Nature remains neutral with regard to jurisdictional claims in published maps and institutional affiliations.

\section{Affiliations}

\section{Fateme Bagherolhashemi ${ }^{1} \cdot$ Mohammad Reza Bozorgmehr $^{1}$ [D $\cdot$ Mohammad Momen-Heravi $^{1}$}

1 Department of Chemistry, Mashhad Branch, Islamic Azad

University, Mashhad, Iran 